EPJ Web of Conferences 41, 03002 (2013)

DOI: $10.1051 /$ epjconf/20134103002

(C) Owned by the authors, published by EDP Sciences, 2013

\title{
Optical and $x$-ray time resolved study of the structural transition in mixed valence manganites
}

\author{
A. Caviezel ${ }^{1}$, U. Staub ${ }^{1}$, S. L. Johnson ${ }^{2}$, S. O. Mariager ${ }^{1}$, G. Ingold ${ }^{1}$, E. Möhr-Vorobeva ${ }^{1}$, M. \\ Garganourakis ${ }^{1}$, S. W. Huang ${ }^{1}$, C. J. Milne ${ }^{3}$, Q. X. Jia ${ }^{4}$, S.-W. Cheong ${ }^{5}$, and P. Beaud ${ }^{1}$ \\ ${ }^{1}$ Swiss Light Source, Paul Scherrer Institut, 5232 Villigen PSI, Switzerland \\ ${ }^{2}$ Institute for Quantum Electronics, Physics Department, ETH Zurich, 8093 Zurich, Switzerland \\ ${ }^{3}$ Laboratoire de Spectroscopie Ultrarapide, EPF Lausanne, 1015 Lausanne, Switzerland \\ ${ }^{4}$ Center for Integrated Nanotechnologies, LANL, Los Alamos, New Mexico 87545, USA \\ ${ }^{5}$ Rutgers Center for Emergent Materials, Rutgers University, Piscataway, NJ 08854, USA
}

\begin{abstract}
Time resolved optical reflectivity and x-ray diffraction techniques are employed to study the laser-induced structural response in two charge and orbitally ordered manganites. Optical data indicate a non-thermal nature of the laser-triggered phase transition via the disappearance of an optical phonon related to the charge and orbitally ordered phase. The x-ray diffraction measurements on superlattice reflections confirm the non-thermal time scale of the initial step of this phase transition but also show that the complete change of structural symmetry is not instantaneous.
\end{abstract}

\section{Introduction}

Transition metal oxides with a perovskite structure feature multifaceted phase diagrams due to their close interaction of charge, atomic order and spin. The high susceptibility to external stimuli, including temperature, magnetic or electric fields, chemical doping and photo-excitation [2-5] yields various ways of manipulating the macroscopic properties in these materials. The most prominent consequence of the close interplay of the different degrees of freedom in manganites is the colossal magnetoresistance effect [1]. The chemical composition $\mathrm{La}_{1-x} \mathrm{Ca}_{x} \mathrm{MnO}_{3}$ (LCMO) is one of the most studied compounds and the $\mathrm{Mn}$ valence varies from truly $3+$ in $\mathrm{LaMnO}_{3}$ to $4+$ in $\mathrm{CaMnO}_{3}$ where the trivalent Mn ion shows a strong Jahn-Teller effect. For a charge carrier concentration of $x>0.5$ the Jahn-Teller effect yields a distortion of the oxygen octahedra at the $\mathrm{Mn}^{3+}$ sites and establishes a superlattice along with a CE-type charge and orbital ordering $(\mathrm{CO} / \mathrm{OO})$ below the ordering temperature $T_{\mathrm{CO}}[6]$. Recent results on a $\mathrm{CO} / \mathrm{OO}$ thin film of $\mathrm{La}_{0.42} \mathrm{Ca}_{0.58} \mathrm{MnO}_{3}$ using femtosecond $\mathrm{x}-$ ray diffraction to probe directly the structural dynamics show that photo-excitation can induce an ultrafast non-thermal phase transition measured via the disappearance of a superlattice reflection [4]. Because the Curie temperature $T_{C}$ is optimized for $x=3 / 8$ in LCMO the system $\mathrm{La}_{5 / 8-y} \mathrm{Pr}_{y} \mathrm{Ca}_{3 / 8} \mathrm{MnO}_{3}$ (LPCMO) is an ideal starting point for further investigations of chemical pressure ranging from $\mathrm{La}_{5 / 8} \mathrm{Ca}_{3 / 8} \mathrm{MnO}_{3}$ with a robust ferromagnetic metallic phase up to $\operatorname{Pr}_{5 / 8} \mathrm{Ca}_{3 / 8} \mathrm{MnO}_{3}$ where a $\mathrm{CO}$ insulating phase is observed [7]. Here, we present a study of the $\mathrm{CO} / \mathrm{OO}$ phase and its behavior upon photo-excitation in a thin LCMO $(x=0.58)$ film and a single crystal of LPCMO $(y=3 / 8)$.

This is an Open Access article distributed under the terms of the Creative Commons Attribution License 2.0, which permits unrestricted use, distribution, and reproduction in any medium, provided the original work is properly cited. 


\section{Experimental}

Optical reflectivity data were collected with a $2 \mathrm{kHz}$ Ti:sapphire laser system delivering $50 \mathrm{fs}$ pulses to an $800 \mathrm{~nm}$ single colour pump-probe setup with cross polarized pump and probe beams to eliminate interferences at time zero and to minimize pump scatter. The setup is equipped with a closed cycle refrigerator allowing temperatures from 5-300 K. A chopper $(1 \mathrm{kHz})$ in the pump branch allows alternate sampling of the excited as well as the unexcited response of the sample. This way electronic drifts of the data collection system can be eliminated.

Time resolved hard x-ray diffraction experiments are performed at the FEMTO slicing beamline at the Swiss Light Source that generates at a repetition rate of $2 \mathrm{kHz} x$-ray pulses of approximately 140 fs duration with a flux of $2 \times 10^{5}$ photons $/ s$ in a $0.1 \%$ bandwidth at $5 \mathrm{keV}$ [8]. A grazing incidence geometry allows matching the $\mathrm{x}$-ray probe depth to the very short absorption lengths of the optical pump beam. The energy of the focused x-ray beam $\left(250 \times 10 \mu \mathrm{m}^{2}\right)$ is selected via a multilayer mirror with a bandwidth of $1.2 \%$ to either $7.1 \mathrm{keV}$ in the LCMO experiment or $5.0 \mathrm{keV}$ for the LPCMO experiment, respectively. The latter energy lies below the $\mathrm{Mn} K$ - and $\operatorname{Pr} L$-edges thus minimizing fluorescence. The sample is excited with a weakly focused laser pulse at $1 \mathrm{kHz}$ at a grazing angle of $10^{\circ}$.

\section{Results and Discussion}

Time resolved optical reflectivity transients are collected for both samples over a broad range of temperatures, 5-300 K, and excitation fluences ranging from 0.5 up to $14 \mathrm{~mJ} / \mathrm{cm}^{2}$. The temperature dependences reproduce well the main findings of previously reported data recorded with high repetition rate lasers $[10,11]$ : A displacively excited coherent phonon with a period of $\sim 2.0 \mathrm{THz}$ in LCMO and $\sim 2.5 \mathrm{THz}$ in LPCMO, only present in the charge and orbitally ordered phase for $T \leq T_{C O}$ $\approx 210 \mathrm{~K}$. There is, however, no sign of a threshold for the reported irreversible melting of the charge order above $20 \mu \mathrm{J} / \mathrm{cm}^{2}$ in LCMO [10].

Figure 1 shows excitation fluence dependent reflectivity changes at $140 \mathrm{~K}$ in LCMO and in LPCMO. For both samples the induced change in reflectivity and the coherent phonon amplitude increase linearly at low fluences. At higher fluences the reflectivity change saturates and the oscillations observed in the optical transient disappear at $\sim 4 \mathrm{~mJ} / \mathrm{cm}^{2}$ for LCMO and $\sim 10 \mathrm{~mJ} / \mathrm{cm}^{2}$ for LPCMO. Since the temperature dependence relates this phonon to the $\mathrm{CO} / \mathrm{OO}$ phase this behaviour may suggest an immediate melting of the underlying ordered phase. Indeed in a recent paper the laser induced disappearance of an optical phonon has been interpreted as a direct consequence of an instantaneous change of structural symmetry [12].

X-ray diffraction directly probes the long range symmetry of a material. The charge and orbital order leads to a doubling of the unit cell and therefore to additional weak reflections. The intensity of these so called superlattice peaks are a measure of the lattice distortion and thus for the magnitude of
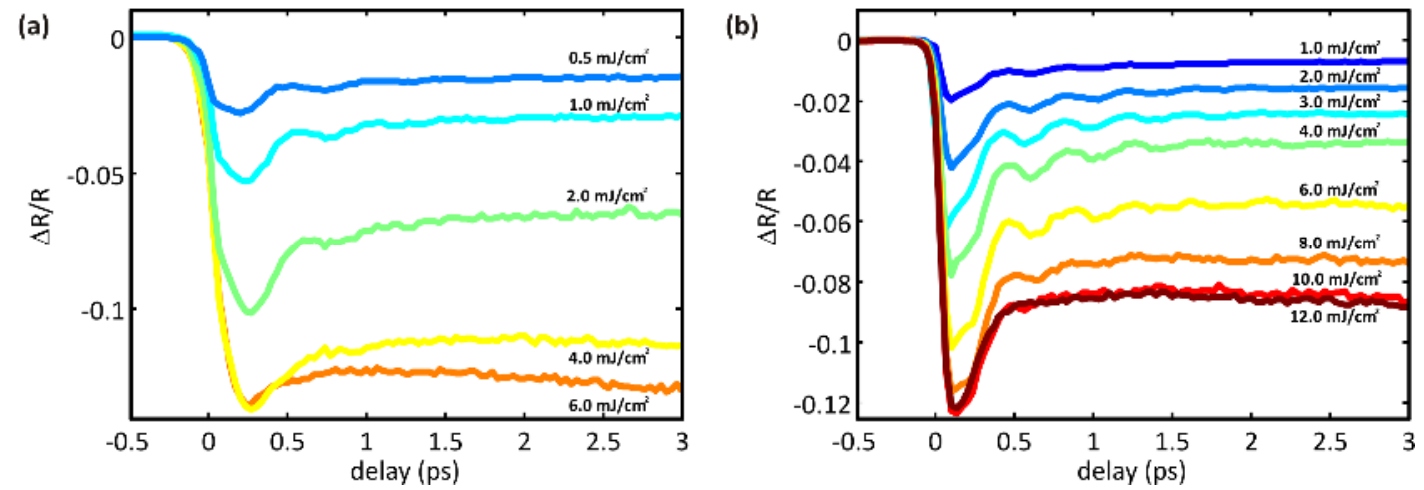

Fig. 1. Measured reflectivity transients at $140 \mathrm{~K}$ for different laser fluences for (a) LCMO and (b) LPCMO. 
(a)

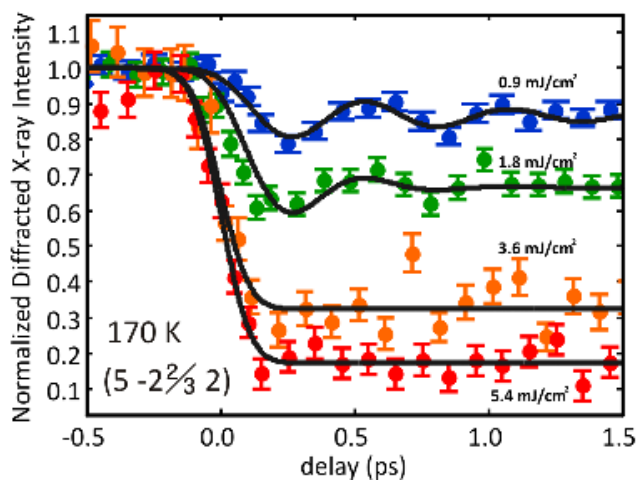

(b)

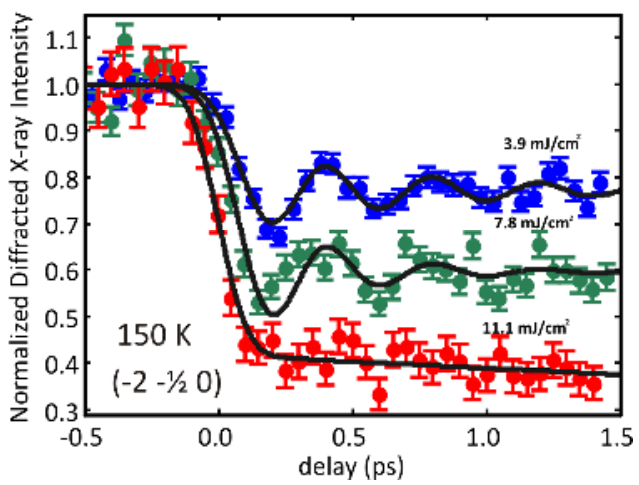

Fig. 2. Time dependences of the normalized diffracted intensity of a superlattice peak are shown for several fluences: (a) LCMO (5 -2 2/3 2) and (b) in LPCMO (-2 -1/2 0).

the ordered phase. The time dependent intensity of superlattice reflections measured for both samples are shown in Figure 2. At low excitation fluences we observe the coherent optical $A_{g}$ phonon also seen in the optical transients. At the pump fluences at which the oscillations vanish in the optical data the x-ray transients feature an immediate drop of roughly $80 \%$ for LCMO and $60 \%$ for LPCMO only limited by the experimental time resolution. At this fluence the complete disappearance of the superlattice peak and therefore a change of structural symmetry occurs on a slower picosecond time scale [4]. The slow decay after the initial drop observed at the highest fluence in Fig. 2 b clearly hints to this slower component. The observed fast drop in superlattice peak intensity has been attributed to the collapse of the Jahn-Teller distortion at the $\mathrm{Mn}^{3+}$ sites following instantaneous disorder introduced in the orbital order by the laser excited intra-atomic $\mathrm{Mn}^{3+}(d-d)$ transition. The partial survival of the SL peaks on a short time scale demonstrates that the change of symmetry does not happen instantaneously as the disappearance of the phonon might imply.

\section{References}

1. G. H. Jonker and J. H. Van Santen, Physica 16, 337 (1950)

2. M. Fiebig, K. Miyano, Y. Tomioka and Y. Tokura, Appl. Phys. B 71, 211 (2000).

3. M. Rini, R. Tobey, N. Dean, J. Itatani, Y. Tomioka, Y. Tokura, R. W. Schoenlein, and A. Cavalleri., Nature 449, 72 (2007).

4. P. Beaud, S. L. Johnson, E. Vorobeva, U. Staub, R. A. De Souza, C. J. Milne, Q. X. Jia, and G. Ingold, Phys. Rev. Lett. 103, 155702 (2009)

5. M. Först, C. Manzoni, S. Kaiser, Y. Tomioka, Y. Tokura, R. Merlin, and A. Cavalleri, Nature Phys. 7, 854 (2011).

6. Y. Tokura and N. Nagaosa, Science 288, 462-468 (2000)

7. M. Uehara, S. Mori, C. H. Chen and S.-W. Cheong, Nature 399, 560-563 (1999)

8. P. Beaud, S. L. Johnson, A. Streun, R. Abela, D. Abramsohn, D. Grolimund, F. Krasniqi, T. Schmidt, V. Schlott, and G. Ingold, Phys. Rev. Lett. 99, 174801 (2007)

9. S. L. Johnson, P. Beaud, E. Vorobeva, C. J. Milne, É. Murray, S. Fahy, and G. Ingold, Acta Cryst. A 66, 157-167 (2010)

10. D. Lim, V. K. Thorsmølle, R. D. Averitt, Q. X. Jia, M. J. Graf, K. H. Ahn, S. A Trugman, and A. J. Taylor, Phys. Rev. B 71, 134403 (2005)

11. K.-J. Jang, J. Lim, J. Ahn, J.H. Kim, K.-J. Yee, and J. S. Ahn, Phys. Rev. B 81, 214416 (2010)

12. S. Wall, D.Wegkamp, L. Foglia, K. Appavoo, J. Nag, R. F. Haglund, J. Stähler, and M. Wolf, Nat. Commun. 3, 721 (2012) 